\title{
Effect of RGD coupled MDA-7/IL-24 on apoptosis induction in a hepatocellular carcinoma cell line
}

\author{
EBRAHIM HOSSEINI $^{1}$, SEYED YOUNES HOSSEINI ${ }^{2}$, TAYEBEH HASHEMPOUR ${ }^{3}$, \\ MOHAMMAD-REZA FATTAHI ${ }^{4}$ and MAJID SADEGHIZADEH ${ }^{1}$
}

\footnotetext{
${ }^{1}$ Department of Molecular Genetics, Faculty of Biological Sciences, Tarbiat Modares University, Tehran 1411713116; ${ }^{2}$ Bacteriology and Virology Department, School of Medicine, Shiraz University of Medical Sciences, Shiraz 71348-14336; ${ }^{3}$ Clinical Microbiology Research Center, Namazi Hospital, Shiraz University of Medical Sciences, Shiraz 71348-45794;

${ }^{4}$ Gastroenterohepatology Research Center (GEHRC), Shiraz University of Medical Sciences, Shiraz 71937-11351, Iran
}

Received July 16, 2015; Accepted November 2, 2016

DOI: 10.3892/mmr.2016.6009

\begin{abstract}
The melanoma differentiation-associated gene-7 (MDA-7) gene, also termed interleukin-24 (IL-24), is a tumor suppressor gene that induces apoptosis in a broad scope of malignant neoplastic cells. The apoptosis induction capacity of the MDA-7/IL-24 gene is partially associated with adhering to cognate receptors. The current study aimed to enhance the antitumor effect of IL-24. The intrinsic signal sequence of IL-24 replaced with a fused artificial signal (secrecon)-RGD4C sequence and its impact was evaluated in HepG2 cells. The modified SP.RGD.IL-24 and native IL-24cDNA sequences were cloned into the pcDNA3.1 expression vector. Subsequently, the expression level, secretion efficacy and targeting propensity of the modified SP.RGD.IL-24 product compared with normal IL-24 by were determined by enzyme-linked immunosorbent assay. The constructs were then transfected into HepG2 and LX-2 cells as tumor and normal hepatic cell lines, respectively. The expression level of the pro-apoptotic DNA damage inducible transcript 3 (Gadd153) and BCL2 associated X apoptosis regulator (Bax) genes in the different groups were compared by reverse transcription-quantitative polymerase chain reaction. Additionally, the rate of apoptosis induction of modified and intact IL-24 sequences was compared by flow cytometry analysis of cells following their propidium iodide/annexin $\mathrm{V}$ staining. SP.RGD-IL-24 protein was expressed and secreted
\end{abstract}

Correspondence to: Professor Majid Sadeghizadeh, Department of Molecular Genetics, Faculty of Biological Sciences, Tarbiat Modares University, 116 Jalal-Al-Ahmad Street, Tehran 1411713116, Iran

E-mail: sadeghma@modares.ac.ir

Dr Seyed Younes Hosseini, Bacteriology and Virology Department, School of Medicine, Shiraz University of Medical Sciences, 21 Zand Boulevard, Shiraz 71348-14336, Iran

E-mail: hoseiniy@sums.ac.ir

Key words: MDA-7, IL-24, RGD peptide, apoptosis, hepatocellular carcinoma in a similar manner to native IL-24, however, the modified SP.RGD.IL-24 adhered to tumor cells more efficiently than IL-24 (P<0.05). SP.RGD.IL-24 significantly induced upregulation of Gadd153 and Bax in HepG2 cells compared with native IL-24 $(\mathrm{P}<0.05)$. However, neither had a significant impact on the expression level of pro-apoptotic genes in LX-2 cells. Flow cytometry analysis also indicated that modified SP.RGD.IL-24 induced apoptosis more than native IL-24 in HepG2 cells $(\mathrm{P}<0.05)$. In conclusion, the novel generated RGD-coupled IL-24 construct exhibited sufficient anticancer activity compared with the native IL-24. The results of the current study provide novel insights for the future of cytokine targeting and indicates its potential capacity as a valuable candidate for gene therapy methods.

\section{Introduction}

Melanoma differentiation-associated gene-7 (MDA-7), also termed interleukin-24 (IL-24), as one of the IL-10 gene family members, exhibits profound anticancer toxicity, with no adverse effect on normal cells (1). The ectopic overexpression of MDA-7/IL-24, either by a plasmid or a recombinant adenovirus (Ad.MDA-7), induces apoptosis in a wide range of tumor cells, however normal cells are not affected (1-3).

Previous studies have demonstrated that the human MDA-7/IL-24 protein performs physiological functions through interacting with two heterodimeric cytokine receptor complexes, IL-20 receptor 1 (IL-20R1)/IL-20R2 and IL-22R1/IL-20R2 (4,5). Unlike the mammalian-produced protein, adenoviral and bacterial-synthesized MDA-7/IL-24, with glutathione S-transferase (GST) tag (GST-IL-24), suppresses tumor cell growth and promotes apoptosis in an IL-20 receptor-independent manner (5). It has been suggested previously that cancer cells may internalize GST-MDA-7/IL-24 independent of the receptor attachment $(6,7)$. By contrast with the GST-MDA-7/IL-24 and Ad.MDA-7 protein products, the purified mammalian-made MDA-7/IL-24 protein did not exert any toxic effect on cells lacking IL-20 receptors $(8,9)$. Thus, IL-24 exhibits its death-inducing function partly via a receptor-dependent pathway as a classical cytokine (in the case of secreted soluble protein), but also via an 
intracellular non-receptor-mediated manner (in the case of GST-MDA-7/IL-24 and Ad.MDA-7) (5).

Theoretically, specific targeting of IL-24 protein to tumor tissues/cells may significantly enhance its antitumor effect in vivo. The arginine-glycine-aspartic acid (RGD) peptide is a conserved motif on certain ligands with affinity to integrins (10). The $\alpha v \beta 3$ integrin is significantly overexpressed in angiogenic endothelial cells and certain tumor cells, such as melanoma, breast cancer, prostate cancer and hepatocellular carcinoma. Since the $\alpha v \beta 3$ integrin is a marker for neovascularization, it has been developed as an encouraging target for cancer therapy $(11,12)$. The phage display technique has demonstrated that the ACDCRGDCFCG (RGD-4C) peptide sequences selectively bind to the $\alpha v \beta 3$ integrin $(13,14)$. Because of the specific interaction between RGD sequence and integrins, targeting delivery of the RGD fused cytokine has been exhibited as an encouraging antineoplastic approach (15-17).

In this regard, it is hypothesized that in comparison with the native form, the RGD coupled IL-24 sequence may harbor an enhanced antitumor activity as it can bind receptors on neighbor cells following secretion. This modification retains the natural intracellular activity of IL-24, but also improves its bystander effect via protein attachment to neighboring cell receptors (18). Accordingly, the current study constructed a novel plasmid vector expressing RGD fused to IL-24 with the aim of improving tumor targeting, then its efficacy was evaluated in vitro. HepG2 hepatocellular carcinoma line and LX-2 human liver stellate cell were selected as tumor and normal cells, respectively, to compare apoptosis induction properties of the modified and the native IL-24.

\section{Materials and methods}

Cell lines and culture. LX-2 human liver stellate cell line was provided by Dr Scott L. Friedman (Mount Sinai School of Medicine, NY, USA). The cell line is characterized as an immortal, non-malignant cell line that retains key features of the hepatic stellate lineage. It was included in the current study as a negative control/normal cell. The HepG2 human liver cancer cell line and Ad-293 cell line were purchased from the National Cell Bank, Pastor Institute of Tehran (Tehran, Iran). All cells were maintained in Dulbecco's modified Eagle's medium containing 10\% fetal bovine serum, $10 \mathrm{mM}$ glutamine, $100 \mathrm{U} / \mathrm{ml}$ penicillin, and $100 \mu \mathrm{g} / \mathrm{ml}$ streptomycin (Thermo Fisher Scientific, Inc., Waltham, MA, USA) under $5 \% \mathrm{CO}_{2}$ atmosphere and $37^{\circ} \mathrm{C}$ condition.

Construction of $p c D N A 3.1 / R G D-I L-24$. The original MDA-7 plasmid (pGEX-5X1/GST-IL-24) was a gift from Dr Stephanie Kreis (Laboratoire de Biologie et Physiologie Integree University of Luxembourg, Esch-sur-Alzette, Luxembourg) (19). The plasmids, expressing IL-24 and SP.RGD.IL-24, were constructed by stepwise cloning. Using the P1 and P2 primers, which harbored the BamHI and XhoI recognition sites (Table I), the normal MDA-7/IL-24 was amplified by Taq DNA polyemerase (Thermo Fisher Scientific, Inc.) using the following cycling conditions: Denaturation at $95^{\circ} \mathrm{C}$ for $5 \mathrm{~min}$; followed by 35 cycles at 95,58 , and $72^{\circ} \mathrm{C}$ for $15 \mathrm{sec}, 40 \mathrm{sec}$, and $1 \mathrm{~min}$, respectively. The amplified segment was directly cloned into the pcDNA3.1 (Addgene, Inc., Cambridge, MA, USA) expression vector. The resulting vector was designated as pc/IL-24.

The modified IL-24 coding sequence with proceeding RGD4C sequence was constructed by replacing the intrinsic IL-24 signal peptide sequence with a fusion of the artificial signal peptide RGD4C sequence. The secrecon is a bioinformatically-designed signal sequence with a high secretion potency, which is composed of 21 amino acids (20). The cleavage site of the secrecon in the fusion sequence was predicted just before the RGD sequence using SignalP v.4.0 software (http://www.cbs.dtu.dk/services/SignalP/) (21).

The fusion segment of the artificial signal peptide RGD4C was made by extending the forward (S1) and the reverse (S2) primers. This sequence was termed SP-RGD4C. The S1 and S2 primers had an overlapping complementary 20-base sequence in their 3' ends (double underline), which allows the primers to extend each other. Additionally, the IL-24 coding region was amplified without its intrinsic signal peptide sequence by the P2 and P3 primers. As the forward primer (P3) was designed immediately following the signal sequence, the amplified segment did not contain the signal sequence. The S2 primer had 25 overlapped bases in the 5 'end (underlined) with the IL-24 sequence immediately following the signal sequence. Therefore, the IL-24 without intrinsic signal sequence was fused to the SP-RGD4C using the synthesis by overlap extension polymerase chain reaction (PCR) method. The total sequence was termed SP.RGD. IL-24. Due to the features of the S1 and P2 primers with the BamHI and the XhoI recognition sites, respectively, SP.RGD. IL-24 final amplicons were adjustable for the directed cloning into the pcDNA3.1 expression vector. The vector, containing the SP.RGD.IL-24 sequence, was termed pc/SP.RGD.IL-24. The gene insertion and the integrity of the constructs were assessed through PCR, restriction analysis and sequencing.

DNA transfection. Cells were plated 1 day before transfection. A total of $4 \times 10^{5}$ cells were seeded per well in 6-well plates and transfected with $1 \mu \mathrm{g}$ plasmid vectors using Lipofectamine-LTX ${ }^{\mathrm{TM}}$ (Thermo Fisher Scientific, Inc.) transfection reagent according to the manufacturer's instructions. The cell lines were transfected separately with empty pCDNA3.1 (pc), pc/IL-24 or pc/SP.RGD.IL-24. To optimize plasmid transfection method and estimate transfection rate, a control GFP-expressing plasmid (pAdenovator-CMV5-IRES-EGFP vector; Qbiogene; MP Biomedicals, LLC, Santa Ana, CA, USA) was also included. The percentage of GFP expression was observed using fluorescent microscopy and used to estimate the transfection rate.

Enzyme-linked immunosorbent assay (ELISA). In order to evaluate the gene expression potency of the new constructs, after cultivating the transfected and non-transfected Ad-293 for $72 \mathrm{~h}$, the supernatants were collected and centrifuged at $3,000 \times \mathrm{g}$ for $5 \mathrm{~min}$ at room temperature. The concentrations of IL-24 or SP.RGD.IL-24 in Ad-293 supernatants were quantified using an IL-24 human ELISA kit (Abcam, Cambridge, MA, USA; cat. no. ab171345), following the manufacturer's instructions. All the experiments were performed in triplicate and the mean value was included in the analysis. 
Cell attachment screening. As the final product of the novel SP.RGD.IL-24 sequence was proposed to anchor to integrin receptors, a simple attachment assay was performed to determine the potency. For this purpose, an ELISA-based method was performed. In this regard, at first Ad-293 cells were transfected with each of the pc.1/IL-24 or pc/SP.RGD. IL-24 constructs. After $72 \mathrm{~h}$, the supernatants of these cells were collected and the concentrations of the gene products were estimated using an IL-24 ELISA kit. The gene products were then diluted to the same concentrations. HepG2 cells were plated in a 6 -well plate $\left(4 \times 10^{5}\right.$ cells per well), 2 days prior to the attachment assay. The HepG2 cell is among the types of liver cell to express integrins, thus, it can support the attachment of RGD-modified protein.

Subsequently, the supernatant of cells was removed and the plated cells were washed twice with PBS. Then, different supernatants from transfected Ad-293 cells, containing different gene products, but with the same concentration, were added to each well of HepG2 cells. After $2 \mathrm{~h}$, the supernatants were removed slowly and the concentrations of the protein products were estimated by the IL-24 ELISA kit again. The reduced amount of the protein concentrations were estimated as the cell attachment capability of the gene products. The assays were performed in triplicate.

cDNA synthesis and quantitative PCR ( $q P C R)$. Total cellular RNA was isolated from cells $36 \mathrm{~h}$ after transfection by using the Total RNA Isolation System kit (Promega Corporation, Madison, WI, USA) in accordance with the manufacturer's instructions. The relative RNA integrity was, then, checked by visualizing the ribosomal RNA bands via gel electrophoresis and using a NanoDrop (Thermo Fisher Scientific, Inc., Pittsburgh, PA, USA). Total RNA $(\sim 1 \mu \mathrm{g})$ was subjected to reverse transcription using the cDNA Synthesis Premix (GeneAll Biotechnology Co. Ltd, Seoul, South Korea), according to the manufacturer's instructions.

qPCR was performed on the ABI 7500 Sequence Detection System (Applied Biosystems; Thermo Fisher Scientific, Inc.) using qPCR SYBR GreenMaster mix (Jena Bioscience GmbH, Jena, Germany). Thermal cycling was performed using the following conditions: $95^{\circ} \mathrm{C}$ for $5 \mathrm{~min}$ as the first denaturation step, followed by 40 cycles at 95,60 , and $72^{\circ} \mathrm{C}$ for $15 \mathrm{sec}, 40 \mathrm{sec}$, and $1 \mathrm{~min}$, respectively. Each individual run was followed by a melting curve analysis for $65-95^{\circ} \mathrm{C}$ to ensure the homogeneity of the PCR products. The phosphoglycerate kinase 1 gene was used as a reference gene to normalize the expression levels. Based on the similar efficiency of the qPCRs, the relative quantification of genes was measured using the $2^{-\Delta \Delta C q}$ method and represented as fold change in expression (22). The assays were performed in triplicate independently. The primer sequences, employed in the qPCR assays, are listed in Table I.

Apoptosis analysis. An apoptosis assay was performed using a propidium iodide (PI)/annexin V-APC staining kit (eBioscience, Inc., San Diego, CA, USA), according to the manufacturer's protocols. In brief, the transfected cells were trypsinized and harvested $48 \mathrm{~h}$ after transfection, washed with PBS, and resuspended in $1 \%$ binding buffer $(200 \mu \mathrm{l})$. The cells of different wells were, then, aliquoted equally and stained sequentially with annexin V-conjugated APC and PI for 15 min
Table I. Primers used in the study.

\begin{tabular}{|c|c|}
\hline Primer name & Sequence $\left(5^{\prime}-3^{\prime}\right)$ \\
\hline P1 & $\begin{array}{l}\text { CCCCCGGATCCGCCATGAATTTTCAACA } \\
\text { GAG }^{\mathrm{a}}\end{array}$ \\
\hline $\mathrm{P} 2$ & $\begin{array}{l}\text { GGGGCTCGAGTCAGAGCTTGTAGAATT } \\
\text { TCT }^{\mathrm{b}}\end{array}$ \\
\hline P3 & GGGGCCCAGGGCCAAGAATTC \\
\hline $\mathrm{S} 1$ & $\begin{array}{l}\text { TTTGGATCCATGTGGTGGAGACTGTGGT } \\
\text { GGCTGCTTCTGTTGCTGCTTCTGTTGTG } \\
\text { GCCTATGGTGTGGGCT }^{\mathrm{c}}\end{array}$ \\
\hline S2 & 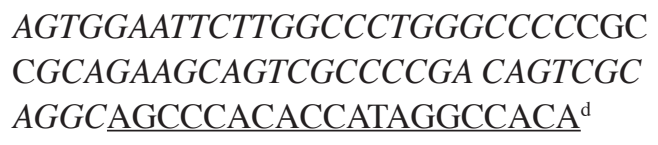 \\
\hline Bax F1 & GCCCTTTTGCTTCAGGGTTTCA \\
\hline Bax R1 & CAGCTTCTTGGTGGACGCAT \\
\hline Gadd153 F & САCCTCCTGGAAATGAAGAGGAAG \\
\hline Gadd 153 R & GAGGTGCTTGTGACCTCTGC \\
\hline PGK1 F1 & TAAAGCCGAGCCAGCCAAAA \\
\hline PGK1 R1 & СТССТАCCATGGAGCTGTGG \\
\hline
\end{tabular}

Underlined sequence represents ${ }^{\mathrm{a}} \mathrm{BamHI}$ and ${ }^{\mathrm{b}} \mathrm{XhoI}$. 'Underlined sequence represents the $B a m H I$ recognition site, double-underlined sequence represents the overlapping complementary sequence, and the italic section represents the signal sequence (secrecon). ${ }^{\mathrm{d}}$ Underlined sequence represents the overlapped base with the interleukin-24 sequence just after the signal sequence, double-underlined sequence represents the overlapping complementary sequence, and the italic section represents the RGD4C sequence. F, forward; R, reverse; Bax, BCL2 associated X apoptosis regulator; Gadd153, DNA damage inducible transcript 3; PGK1, phosphoglycerate kinase 1.

in the dark. Subsequently, $300 \mu \mathrm{l}$ binding buffer was added to the mixture and the acquisition was immediately performed on a FACSCalibur flow cytometer (BD Biosciences, Franklin Lakes, NJ, USA). The analyses were completed by using Cell Quest Pro 5.1 software package (BD Biosciences). The sum of early and late apoptosis cells percentages was considered as the total percentage of apoptotic cells for further analysis. The assays were performed in three independent experiments and subsequently, the means of each group were statistically compared.

Statistical analysis. In all of the experiments, the statistical differences between the means were evaluated by one-way analysis of variance followed by Tukey post test evaluation. $\mathrm{P}<0.05$ was considered to indicate a statistically significant difference. Values are presented as the mean \pm standard deviation.

\section{Results}

Construction and expression of pcDNA3.1/IL-24 and pcDNA3.1/SP.RGD.IL-24. Following cloning of two different sequences into the pcDNA3.1 plasmid, all PCR reactions, restriction analysis and sequencing result confirmed the vector integrity, sequence accuracy, and the correct direction of 


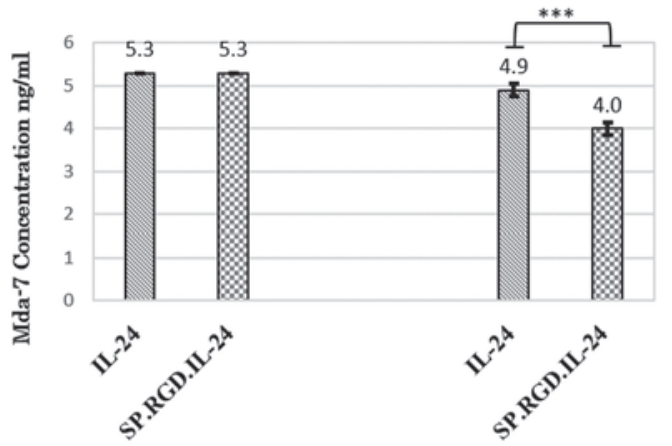

Figure 1. Cell attachment assay of the modified SP.RGD.IL-24 product. The concentrations of IL-24 and SP.RGD.IL-24 products in the supernatant before culture with HepG2 cells (left), and after (right). The concentration decrease, following the treatment of HepG2 cells, was considered to indicate the cell attachment ability of SP.RGD.IL-24 protein, compared with the native IL-24 $(\mathrm{P}<0.05)$. The native IL-24 product was used as the assay control. The numbers above each columns represent the mean of the three different experiments, and the bars display the standard deviation. IL-24, interleukin-24. ${ }^{* * *} \mathrm{P}<0.05$, comparison indicated by brackets.

inserts. The sequencing results confirmed that SP.RGD.IL-24 had an artificial signal sequence, followed by the RGD4C sequence, and the sequence of IL-24 beyond its intrinsic signal sequence.

The IL-24 gene, with an intrinsic signal peptide, produces a secretory protein, which is a common feature of all cytokines. Additionally, the novel SP.RGD.IL-24 sequence, with an artificial signal peptide, was expected to similarly produce a secretory protein. As the most reliable test for the protein secretion assay, the concentration of IL-24 in the supernatants of the transfected cells was quantified by ELISA. The results demonstrated that the concentration of IL-24 in the supernatant of the A-293 cells transfected with pc/SP.RGD.IL-24, was fairly similar to pc/IL-24, albeit the former construct exhibited less production $(\mathrm{P}>0.05)$. As expected, the concentration of IL-24 in the supernatant of the transfected cells, with an empty pcDNA3.1, was negligible (data not shown).

Tethered RGD motif targets IL-24 to cancer cells. To assess whether the RGD motif of SP.RGD.IL-24 is functional and if is accessible to its cognate integrin, $\alpha v \beta 3$, an ELISA based method was employed. Reduction of gene products in the presence of HepG2 cells indirectly indicated that adhesion of SP.RGD.IL-24 to integrin $\alpha v \beta 3$ is increased compared with IL-24 (Fig. 1).

Effect of the modified SP.RGD.IL-24 on the expression level of pro-apoptotic DNA damage inducible transcript 3 (Gadd153) and BCL2 associated X apoptosis regulator (Bax) genes. Three prepared plasmids were transfected into LX-2 cells (as the normal control) and HepG2 liver cancer cells. Ad-293, LX-2 and HepG2 cells exhibited different rates of susceptibility to transfection. The GFP signal counting under microscope (data not shown) indicated that transfection rate for Ad-293, LX-2 and HepG2 cells were estimated to be $\sim 80$, 70 and 50\% respectively, during experiments. Subsequently, qPCR was employed to compare the expression level of two pro-apoptotic genes (Gadd153 and Bax), following transfection of plasmids expressing IL-24 and the modified SP.RGD.
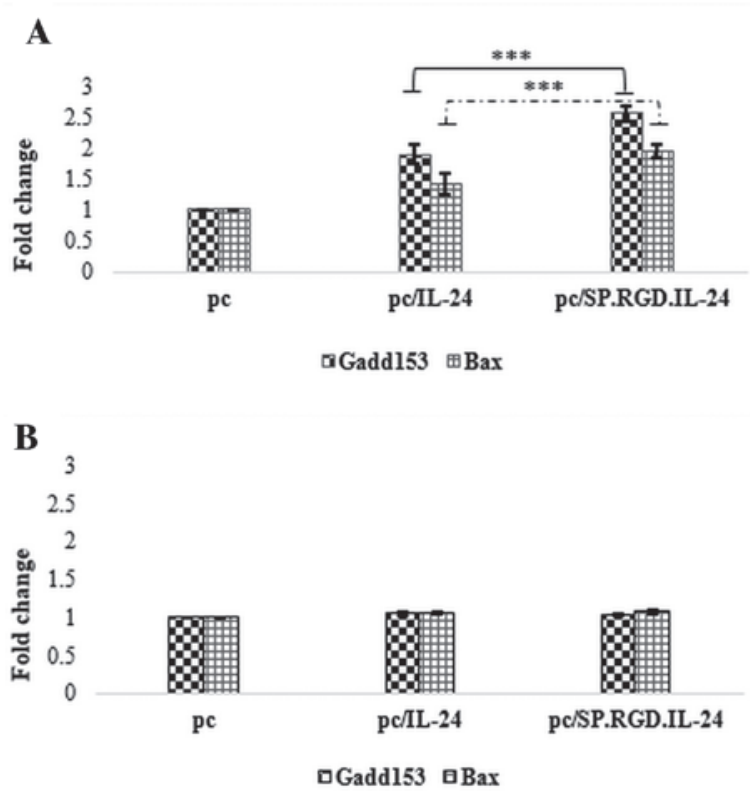

Figure 2. Effect of IL-24 and the modified SP.RGD.IL-24 on the expression level of Gadd153 and Bax pro-apoptotic genes in HepG2 and LX-2 cells. Gadd153 and Bax mRNA expression levels were evaluated by reverse transcription-quantitative polymerase chain reaction relative to the phosphoglycerate kinase 1 as a reference gene, in HepG2 and LX-2 cells, $36 \mathrm{~h}$ after the transfection with pcDNA3.1, pc/IL-24 or pc/SP.RGD.IL-24 plasmids. (A) Gadd153 and Bax mRNA expression levels in HepG2 cells, transfected with either pc/IL-24 or pc/SP.RGD.IL-24 plasmids were significantly higher $(\mathrm{P}<0.05)$ than the cells transfected with pcDNA3.1. Gadd153 and Bax mRNA expression levels in HepG2 cells, transfected with pc/SP.RGD.IL-24 plasmids were higher (74 and $73 \%$, respectively) than the cells, transfected with pc/IL-24. (B) Gadd153 and Bax mRNA expression levels in LX-2 cells, transfected with pcDNA3.1, pc/IL-24 or pc/SP.RGD.IL-24 plasmids did not exhibit significant differences $(\mathrm{P}>0.05)$. The columns represent the means of three different experiments, and the bars indicate the standard deviation. ${ }^{* * *} \mathrm{P}<0.05$, comparison indicated by brackets. pc, pcDNA3.1; IL-24, interleukin-24; Gadd153, DNA damage inducible transcript 3; Bax, BCL2 associated $\mathrm{X}$ apoptosis regulator.

IL-24 in LX-2 and HepG2 cells. Notably, the attained data exhibited a significant difference of the response to plasmids in normal and tumor cells. The expression data analysis demonstrated that the expression of the modified SP.RGD. IL-24, similarly to IL-24, significantly upregulated the Gadd153 (2.5 fold) and Bax (1.9 fold) expression levels in RNA extracted from plasmid-transfected LX-2 and HepG2 cells, compared with the empty plasmid when assayed on HepG2 $(\mathrm{P}<0.05)$. However, the gene expression induced by the SP.RGD.IL-24 construct was significantly greater than that induced by IL-24. As a result, Gadd153 and Bax exhibited 74 and $73 \%$ overexpression, respectively $(\mathrm{P}<0.05)$. In contrast to the HepG2 cells, the expression of IL-24 or SP.RGD.IL-24 in LX-2 cells did not have a significant effect on Gadd153 and Bax mRNA expression levels compared with the empty plasmid ( $\mathrm{P}>0.05$; Fig. 2).

Apoptosis induction by the modified SP.RGD.IL-24. The annexin/PI method was used to investigate the apoptosis induction of different constructs by flow cytometry. As a result, in the analysis, the Q2 and Q3 quadrants were determined as the early and late apoptosis-induced cells, respectively (Fig. 3A). The percentage of total apoptosis (early and late stages) for 
the acquired population following HepG2 transfection were as follows: $19.8 \pm 4.6 \%$ for the native IL-24 $(\mathrm{P}=0.00051)$, $27.5 \pm 4.8 \%$ for the modified SP.RGD.IL-24, and $3.9 \pm 1.4 \%$ for the empty plasmid (Fig. 3). Modified SP.RGD.IL-24 had significantly higher apoptosis induction potency when compared with unmodified IL-24 $(\mathrm{P}<0.05)$. Additionally, the amount of necrosis events was negligible according to the data from the Q1 section (Fig. 3A). In transfected LX-2 cells, no significant changes in the apoptotic population was detected compared with the control group, and the quantity of cells in Q2 and Q3 was similar ( $<4 \%)$ in all groups (Fig. 3).

In brief, the results demonstrated that the modified SP.RGD.IL-24 was more efficient in the induction of apoptosis in HepG2 cells compared with the IL-24. However, SP.RGD. IL-24, similar to IL-24, had no detectable effect on apoptosis of non-cancerous LX-2 cells.

\section{Discussion}

It remains unclear how MDA-7/IL-24 exerts its cancer-specific cell killing activity. MDA-7/IL-24 predominantly exhibits antitumor activity via ER stress and other intracellular apoptosis pathways. An additional scenario proposes that IL-24 exerts its activity, as a classical cytokine, in an extracellular manner, known as the bystander effect. It was demonstrated that soluble MDA-7/IL-24 acts through a cell signaling pathway by interacting with the IL-20/IL-22 receptor complexes (23). Thus, the primary objective of the current study was to improve the apoptotic properties of IL-24 through combining intracellular activity with its receptor-dependent function, bystander effect. To achieve this an RGD coupled IL-24 sequence was cloned in such a way to perform the apoptosis activity like the native form and to also increase the bystander effect due to an RGD peptide sequence in the $\mathrm{N}$-terminal.

The toxic bystander effect of secreted cytokines may be improved by targeting of cancer cells using peptides. Pei et al inserted a glycine code between glutamic acid and the arginine code of IL-24 to produce a modified RGD-IL-24 with an internal mutated RGD sequence. This modification enhanced the attachment of the product to cancer cells and improved its apoptosis-inducing function (24).

The most effective and established RGD-associated motif is the RGD4C peptide. The sequence of this motif was added to that of the IFN- $\alpha$ gene. Then, the modified IFN- $\alpha$.RGD was introduced into tumor vessels by a DNA plasmid. The modified IFN- $\alpha$.RGD gene therapy exhibited a more effective suppression of tumor development than the wild-type IFN- $\alpha$ gene therapy (17).

In our previous study, it was demonstrated that adding the RGD4C sequence to the carboxyl end of IL-24 adversely decreases its antitumor function. The modeling analysis revealed that this kind of modification strongly disrupts the IL-24 binding to the relevant receptor in silico (25). However, RGD4C motif in the amino-terminal of MDA-7/IL-24 protein, which was produced in bacteria, did not have any effect on its apoptosis-inducing activity (26). On the basis of these findings, a vector expressing modified RGD-IL-24 cDNA with a proceeding RGD4C-coding sequence was constructed.

Even though with limited studies regarding the application of exogenous/soluble IL-24 protein $(24,27)$ a question may

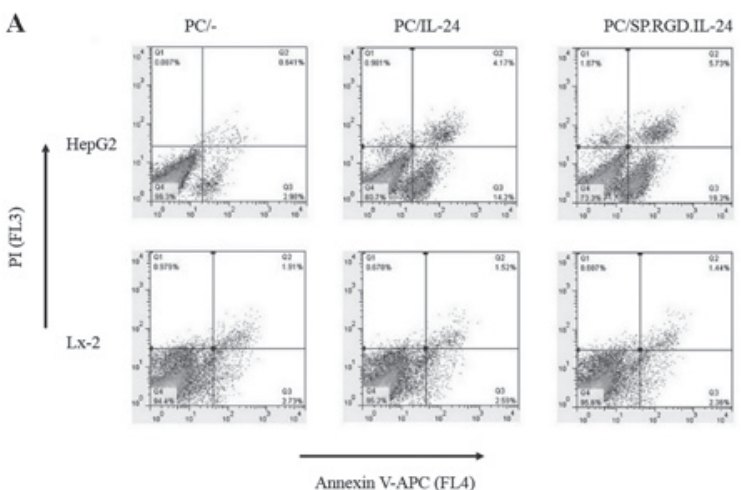

B

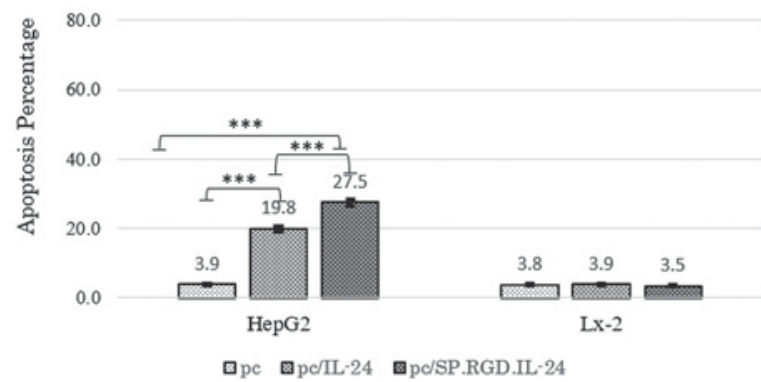

Figure 3. Flow cytometry analysis of apoptosis induction by IL-24 and the modified SP.RGD.IL-24. (A) Analysis of annexin $\mathrm{V}^{+} / \mathrm{PI}^{+} \mathrm{HepG} 2$ and LX-2 cells following transfection with pcDNA3.1, pc/IL-24 or pc/SP.RGD. IL-24 plasmids. Dead cells were scored as necrotic (PI-positive/annexin V-negative, Q1), early apoptotic (PI-negative/annexin V-positive, Q3) and late apoptotic (PI-positive/annexin V-positive, Q2), following a gating using pcDNA transfected cells. Annexin plots (Q2+Q3) illustrated that $48 \mathrm{~h}$ after IL-24 overexpression, the number apoptotic HepG2 cells increased $20 \%$. Overexpression of the modified SP.RGD.IL-24 in HepG2 cells caused a 28\% increase in apoptosis after $48 \mathrm{~h}$. IL-24 or SP.RGD.IL-24 overexpression did not significantly affect the viability of LX-2 cells. (B) Quantification of the obtained data in a column chart; the columns represent the means of three different experiments, the bars represent the standard deviation. ${ }^{* * *} \mathrm{P}<0.05$, comparison indicated by brackets.

arise that why exogenous protein was not used instead of the plasmid vector in the current study? It was demonstrated that direct application of soluble IL-24 protein for tumor killing exhibited less cytotoxic effect, when compared with protein endogenously expressed from a plasmid. This difference is partly related to the more effective interaction of endogenous IL-24 protein with endoplasmic chaperone protein BiP/GRP78, which induces endoplasmic stress (28).

Here, the modified SP.RGD.IL-24 was designed so that the intrinsic signal sequence of IL-24 is exchanged with a fusion of the artificial signal sequence (secrecon) and the RGD4C sequence. Since the signal sequence of IL-24 is long (49 amino acids), a shorter signal sequence with a higher secretion potency was used. As the secrecon, a bioinformatically-designed signal sequence (20), was employed for the first time fused with IL-24, it was not clear if it would retain the secretion property of SP-RGD-IL-24. The ELISA result using the supernatant of the transfected Ad-293 cells demonstrated suitable secretion of the modified RGD-IL-24, indicating the correct action of the secrecon. The expression level of the modified and the native IL-24 were comparable as determined by ELISA. However, the data also indicated that these types of modifications on 
IL-24 cause a relative decrease in protein secretion, albeit not at a significant level.

An ELISA-based assay was also applied to observe if the modified SP.RGD.IL-24 product, compared to the native IL-24, has increased adhesion properties for cancerous HepG2 cells. This method demonstrated that the targeting of SP.RGD. IL-24 to HepG2 cells was increased compared with native IL-24 product.

It was demonstrated that the ectopic expression of IL-24 leads to an enhanced expression of pro-apoptotic genes, including Gadd153 and Bax (29,30). The upregulation of Bax gene expression is a well-established marker of apoptosis with roles in the intrinsic and extrinsic apoptosis pathways, and was thus, selected for expression analysis in the current study (31). Gadd153 is involved in ER stress-associated apoptosis and its expression is known to be induced following endogenous IL-24 expression (6). The ability of the modified SP.RGD.IL-24 and the unmodified IL-24 to induce expression of these pro-apoptotic genes was compared following the transfection of HepG2 cell lines with the IL-24 constructs. qPCR analysis demonstrated that the modified SP.RGD.IL-24 upregulated the expression of pro-apoptotic genes more efficiently than the unmodified IL-24. This finding demonstrated that the RGD modification did have a detrimental impact on the function of the modified IL-24 protein. However, neither the modified SP.RGD.IL-24, nor the native IL-24, had an observable effect on the expression of these pro-apoptotic genes in the LX-2 human liver stellate cell line. To the best of our knowledge, the effect of IL-24 on normal liver stellate cell apoptosis has not been previously investigated. This mode of IL-24 cytokine action on human stellate cell may strongly support its safety prolife for employment in human patients.

Annexin/PI staining and flow cytometry analysis, in accordance with the expression results, revealed that the modified SP.RGD.IL-24, compared with the unmodified IL-24 gene, had a greater capacity to induce apoptosis in the HepG2 cancer cell line. In the vast majority of previous cases, adenovector was employed as the expression/delivery vector of IL-24 in vitro and in vivo. The result of the adenovirus expression vector indicated suitable production of IL-24 and apoptosis induction (33.5\%) (32-35). However, in the present study a plasmid construct was employed to express the modified and native IL-24. The previously obtained results supported the critical role of adenovector expression vector in increasing the induction of apoptosis by IL-24 (32-35). By contrast to adenovectors, plasmid expression of IL-24 is considered less effective and, thus, apoptosis induction decreases. Increased levels of apoptosis are expected when these plasmid constructs are replaced by an adenovector-expression system. However, the modified SP.RGD-IL-24 or native IL-24 did not induce apoptosis in the LX-2 human liver fibroblast cell line.

In conclusion, the findings of the current study indicated the following: i) The new RGD modified construct retained sufficient expression and secretion propensity; ii) SP.RGD-IL-24 attachment to the cognate receptor and integrin was increased compared with native IL-24; and iii) SP.RGD-IL-24 triggered apoptosis in a HCC-associated cell more effectively than native IL-24, and did not affect apoptosis in normal stellate cells. Collectively, the obtained findings supported that the newly generated construct may be utilized in an adenoviral vector as a method of gene therapy.

\section{Acknowledgements}

The authors would like to highly appreciate all the help of the members of the Clinical Microbiology Research Center at Shiraz University of Medical Sciences, especially Dr Mehdi Kalani for her assistance in flow cytometry analysis. The authors also wish to thank other colleagues in the Clinical Microbiology Research Center at Shiraz University of Medical Sciences, including Mr. Javad Moayedi, Ms. Maryam Mousavi, Mr. Saeed Amirzadeh, Ms. Maryam Nejabat and Mr. Amir Arastefar.

\section{References}

1. Ekmekcioglu S, Ellerhorst J, Mhashilkar AM, Sahin AA, Read CM, Prieto VG, Chada S and Grimm EA: Down-regulated melanoma differentiation associated gene (mda-7) expression in human melanomas. Int J Cancer 94: 54-59, 2001.

2. Ellerhorst JA, Prieto VG, Ekmekcioglu S, Broemeling L, Yekell S, Chada S and Grimm EA: Loss of MDA-7 expression with progression of melanoma. J Clin Oncol 20: 1069-1074, 2002.

3. Zhang J, Sun A, Xu R, Tao X, Dong Y, Lv X and Wei D: Cell-penetrating and endoplasmic reticulum-locating TAT-IL-24-KDEL fusion protein induces tumor apoptosis. J Cell Physiol 231: 84-93, 2016.

4. Parrish-Novak J, Xu W, Brender T, Yao L, Jones C, West J, Brandt C, Jelinek L, Madden K, McKernan PA, et al: Interleukins 19, 20, and 24 signal through two distinct receptor complexes. Differences in receptor-ligand interactions mediate unique biological functions. J Biol Chem 277: 47517-47523, 2002.

5. Persaud L, De Jesus D, Brannigan O, Richiez-Paredes M, Huaman J, Alvarado G, Riker L, Mendez G, Dejoie J and Sauane M: Mechanism of Action and Applications of Interleukin 24 in Immunotherapy. Int J Mol Sci 17: pii.E869, 2016.

6. Lebedeva IV, Emdad L, Su ZZ, Gupta P, Sauane M, Sarkar D, Staudt MR, Liu SJ, Taher MM, Xiao R, et al: mda-7/IL-24, novel anticancer cytokine: Focus on bystander antitumor, radiosensitization and antiangiogenic properties and overview of the phase I clinical experience (Review). Int J Oncol 31: 985-1007, 2007.

7. Emdad L, Lebedeva IV, Su ZZ, Gupta P, Sauane M, Dash R, Grant S, Dent P, Curiel DT, Sarkar D and Fisher PB: Historical perspective and recent insights into our understanding of the molecular and biochemical basis of the antitumor properties of mda-7/IL-24. Cancer Biol Ther 8: 391-400, 2009.

8. Nishikawa T, Ramesh R, Munshi A, Chada S and Meyn RE: Adenovirus-mediated mda-7 (IL24) gene therapy suppresses angiogenesis and sensitizes NSCLC xenograft tumors to radiation. Mol Ther 9: 818-828, 2004.

9. Pataer A, Bocangel D, Chada S, Roth JA, Hunt KK and Swisher SG: Enhancement of adenoviral MDA-7-mediated cell killing in human lung cancer cells by geldanamycin and its 17-allyl- amino-17-demethoxy analogue. Cancer Gene Ther 14: 12-18, 2007.

10. Ruoslahti E: The RGD story: A personal account. Matrix Biol 22: 459-465, 2003

11. Lu X, Lu D, Scully M and kakkar V: The role of integrins in cancr and integrin therapeutic agent for cancer therapy. Perspect Medicin Chem 2: 57-73, 2008.

12. Brooks PC, Clark RA and Cheresh DA: Requirement of vascular integrin alpha v beta 3 for angiogenesis. Science 264: 569-571, 1994.

13. Assa-Munt N, Jia X, Laakkonen P and Ruoslahti E: Solution structures and integrin binding activities of an RGD peptide with two isomers. Biochemistry 40: 2373-2378, 2001.

14. Arap W, Pasqualini R and Ruoslahti E: Cancer treatment by targeted drug delivery to tumor vasculature in a mouse model. Science 279: 377-380, 1998.

15. Dickerson EB, Akhtar N, Steinberg H, Wang ZY, Lindstrom MJ, Padilla ML, Auerbach R and Helfand SC: Enhancement of the antiangiogenic activity of interleukin-12 by peptide targeted delivery of the cytokine to alphavbeta3 integrin. Mol Cancer Res 2: 663-673, 2004. 
16. Curnis F, Gasparri A, Sacchi A, Longhi R and Corti A: Coupling tumor necrosis factor-alpha with alphaV integrin ligands improves its antineoplastic activity. Cancer Res 64: 565-571, 2004.

17. Craig R, Cutrera J, Zhu S, Xia X, Lee YH and Li S: Administering plasmid DNA encoding tumor vessel-anchored IFN-alpha for localizing gene product within or into tumors. Mol Ther 16: 901-906, 2008

18. Sauane M, Su ZZ, Gupta P, Lebedeva IV, Dent P, Sarkar D and Fisher PB: Autocrine regulation of mda-7/IL-24 mediates cancer-specific apoptosis. Proc Natl Acad Sci USA 105 9763-9768, 2008

19. Khodadad M, Hosseini SY, Shenavar F, Erfani N, Bina S, Ahmadian S, Fattahi MR and Hajhosseini R: Construction of expressing vectors including melanoma differentiation-associated gene-7 (mda-7) fused with the RGD sequences for better tumor targeting. Iran J Basic Med Sci 18: 780-787, 2015.

20. Barash S, Wang W and Shi Y: Human secretory signal peptide description by hidden Markov model and generation of a strong artificial signal peptide for secreted protein expression. Biochem Biophys Res Commun 294: 835-842, 2002.

21. Petersen TN, Brunak S, von Heijne G and Nielsen H: SignalP 4.0: Discriminating signal peptides from transmembrane regions. Nat Methods 8: 785-786, 2011.

22. Livak KJ and Schmittgen TD: Analysis of relative gene expression data using real-time quantitative PCR and the 2(-Delta Delta C(T)) Method. Methods 25: 402-408, 2001.

23. Dent P, Yacoub A, Hamed HA, Park MA, Dash R, Bhutia SK, Sarkar D, Gupta P, Emdad L, Lebedeva IV, et al: MDA-7/IL-24 as a cancer therapeutic: From bench to bedside. Anticancer Drugs 21: 725-731, 2010.

24. Pei DS, Yang ZX, Zhang BF, Yin XX, Li LT, Li HZ and Zheng JN: Enhanced apoptosis-inducing function of MDA-7/IL-24 RGD mutant via the increased adhesion to tumor cells. J Interferon Cytokine Res 32: 66-73, 2012.

25. Bina S, Shenavar F, Khodadad M, Haghshenas MR, Mortazavi M, Fattahi MR, Erfani N and Hosseini SY: Impact of RGD peptide tethering to IL24/mda-7 (Melanoma Differentiation Associated Gene-7) on apoptosis induction in hepatocellular carcinoma cells. Asian Pac J Cancer Prev 16: 6073-6080, 2015.

26. Xiao B, Li W, Yang J, Guo G, Mao XH and Zou QM: RGD-IL-24, a novel tumor-targeted fusion cytokine: Expression, purification and functional evaluation. Mol Biotechnol 41: 138-144, 2009.
27. Ma Q, Jin B, Zhang Y, Shi Y, Zhang C, Luo D, Wang P, Duan C, Song H, Li X, et al: Secreted recombinant human IL-24 protein inhibits the proliferation of esophageal squamous cell carcinoma Eca-109 cells in vitro and in vivo. Oncol Rep 35: 2681-2690, 2016.

28. Sieger KA, Mhashilkar AM, Stewart A, Sutton RB, Strube RW, Chen SY, Pataer A, Swisher SG, Grimm EA, Ramesh R and Chada S: The tumor suppressor activity of MDA-7/IL-24 is mediated by intracellular protein expression in NSCLC cells. Mol Ther 9: 355-367, 2004.

29. Su ZZ, Madireddi MT, Lin JJ, Young CS, Kitada S, Reed JC, Goldstein NI and Fisher PB: The cancer growth suppressor gene mda-7 selectively induces apoptosis in human breast cancer cells and inhibits tumor growth in nude mice. Proc Natl Acad Sci USA 95: 14400-14405, 1998.

30. Sarkar D, Su ZZ, Lebedeva IV, Sauane M, Gopalkrishnan RV, Valerie K, Dent P and Fisher PB: mda-7 (IL-24) Mediates selective apoptosis in human melanoma cells by inducing the coordinated overexpression of the GADD family of genes by means of p38 MAPK. Proc Natl Acad Sci USA 99: 10054-10059, 2002.

31. Tait SW and Green DR: Mitochondria and cell death: Outer membrane permeabilization and beyond. Nat Rev Mol Cell Biol 11: 621-632, 2010.

32. Wang CJ, Xue XB, Yi JL, Chen K, Zheng JW, Wang J, Zeng JP and Xu RH: Melanoma differentiation-associated gene-7, MDA-7/IL-24, selectively induces growth suppression, apoptosis in human hepatocellular carcinoma cell line HepG2 by replication-incompetent adenovirus vector. World J Gastroenterol 12 1774-1779, 2006.

33. Nishikawa T, Ramesh R, Munshi A, Chada S and Meyn RE: Adenovirus-mediated mda-7 (IL24) gene therapy suppresses angiogenesis and sensitizes NSCLC xenograft tumors to radiation. Mol Ther 9: 818-828, 2004.

34. Zhao L, Gu J, Dong A, Zhang Y, Zhong L, He L, Wang Y, Zhang J, Zhang Z, Huiwang J, et al: Potent antitumor activity of oncolytic adenovirus expressing mda-7/IL-24 for colorectal cancer. Hum Gene Ther 16: 845-858, 2005.

35. Pan X, Wu L, Cao J, Guo W, Wang Z, Han B and Hu W: Recombinant adenovirus vector-mediated human MDA-7 gene transfection suppresses hepatocellular carcinoma growth in a mouse xenograft model. J Biomed Res 26: 53-58, 2012. 